\title{
SLAMF6/Ly108 promotes the development of hepatocellular carcinoma via facilitating macrophage $\mathrm{M} 2$ polarization
}

\author{
QI MENG $^{1 *}$, XIUYUN DUAN $^{1 *}$, QINGCHAO YANG $^{1 *}$, DEWEN XUE ${ }^{2}$, ZIHAO LIU $^{2}$, YUANYUAN LI $^{3}$,

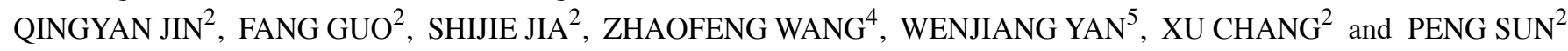 \\ ${ }^{1}$ School of Cheeloo Clinical Medicine, Shandong University, Jinan, Shandong 250102; \\ ${ }^{2}$ Department of Intervention Oncology and ${ }^{3}$ Head and Neck Radiation Oncology Ward II, \\ Shandong Cancer Hospital and Institute, Shandong First Medical University and Shandong Academy of Medical Sciences, \\ Jinan, Shandong 250117; ${ }^{4}$ Surgical Department, Jinan Jiyang District Hospital of Traditional Chinese Medicine, \\ Jinan, Shandong 251499; ${ }^{5}$ The Key Laboratory of Cardiovascular Remodeling and Function Research, \\ Chinese Ministry of Education, Chinese National Health Commission and Chinese Academy of Medical Sciences, \\ The State and Shandong Province Joint Key Laboratory of Translational Cardiovascular Medicine, \\ Department of Cardiology, Qilu Hospital of Shandong University, Jinan, Shandong 250012, P.R. China
}

Received July 27, 2021; Accepted November 24, 2021

DOI: $10.3892 / \mathrm{ol} .2022 .13203$

\begin{abstract}
Tumor-associated macrophages (TAMs) are capable of worsening hepatocellular carcinoma (HCC) prognosis by accelerating tumor growth and progression. Signaling lymphocyte activation molecule family member 6 (SLAMF6; Ly108 in mice) is an immune regulator that is involved in numerous diseases. However, whether SLAMF6 might affect macrophage function in HCC has not yet been reported. Therefore, the present study aimed to determine the relationship between SLAMF6 expression on macrophages and HCC progression. In the present study, the expression of SLAMF6 in human blood samples and mice was analyzed by flow cytometry. Furthermore, macrophage-related polarization markers were detected via reverse transcription quantitative PCR. Clonogenic formation and Transwell assay were performed to
\end{abstract}

Correspondence to: Dr Peng Sun, Department of Intervention Oncology, Shandong Cancer Hospital and Institute, Shandong First Medical University and Shandong Academy of Medical Sciences, 440 Jiyan Road, Jinan, Shandong 250117, P.R. China

E-mail: sunpeng58928@163.com

*Contributed equally

Abbreviations: BMDM, bone marrow-derived macrophage; HCC, hepatocellular carcinoma; IFN- $\gamma$, interferon gamma; IL-6, interleukin-6; LPS, lipopolysaccharide; PM, peripheral macrophage; RNS, reactive nitrogen species; ROS, reactive oxygen species; SLAMF, signaling lymphocytes activation molecule family; SLE, systemic lupus erythematous; TAMs, tumor-associated macrophages; TNF- $\alpha$, tumor necrosis factor- $\alpha$

Key words: hepatocellular carcinoma, signaling lymphocyte activation molecule family member 6/Ly108, M2 macrophage determine the proliferation, migration and invasion of $\mathrm{HCC}$ cells. In addition, a murine HCC model was established to detect the function of SLAMF6 in vivo. The results demonstrated that SLAMF6 expression was increased in $\mathrm{CD} 14^{+}$cells obtained from patients with HCC. It was also determined that this increase was associated with a positive hepatitis B virus DNA status and high levels of $\alpha$-fetoprotein. Polarized TAMs from THP-1 cells, murine peritoneal macrophages and murine bone marrow-derived macrophages all exhibited higher levels of SLAMF6 compared with M1 cells. Furthermore, an increased expression of Ly108 was detected in macrophages obtained from mice tumor tissues, indicating that the tumor microenvironment may promote Ly108 expression and macrophage M2 polarization. Ly108 small interfering RNA was applied to macrophages, which resulted in the suppression of M2 polarization. Ly108-silenced macrophages attenuated HCC cell migration and invasion and prevented tumor growth by inhibiting the nuclear factor- $\kappa \mathrm{B}$ pathway. Altogether, the results from the present study suggested that SLAMF6/Ly108 was upregulated in TAMs, which may in turn accelerate the development of HCC.

\section{Introduction}

Liver cancer was the seventh most common type of cancer and the second leading cause of cancer-associated mortality worldwide in 2020 (1). Among patients with liver cancer, hepatocellular carcinoma (HCC) accounts for $>90 \%$ of cases. Patients with HCC typically demonstrate a poor prognosis, which can be attributed to a diagnosis at an advanced stage (2). Previous studies have reported that immunotherapy can serve an important role in the treatment of $\operatorname{HCC}(3,4)$. Based on these previous studies, it is necessary to evaluate the functional molecules associated with HCC in order to determine a potential therapeutic target in the HCC immune microenvironment. 
Tumor-associated macrophages (TAMs) are a type of primary tumor-infiltrating immune cells (5) that have been demonstrated to promote tumor growth, metastasis and invasion $(6,7)$. TAMs can be divided into two main types as follows: The classically activated M1 phenotype and the alternatively activated M2 phenotype (8). M1 macrophages are polarized by the activation of interferon gamma (IFN- $\gamma$ ), promoting the T-helper (Th)1 immune response and antitumor activity $(9,10)$. M2 macrophages are polarized by interleukin (IL)-4/IL-13 and are associated with the anti-inflammatory $\mathrm{Th} 2$ immune response, which demonstrates a pro-tumor activity (11). Previous studies have revealed that most TAMs are of the M2 phenotype, which promote tumor growth and progression and therefore worsen the prognosis of patients with $\operatorname{HCC}(12,13)$. To further validate the results of these studies, it is necessary to explore and block the key stages of macrophage M2 polarization in order to inhibit HCC progression.

The signaling lymphocyte activation molecule (SLAM) family comprises the following nine members: CD150 (SLAMF1), CD48 (SLAMF2), CD229 (SLAMF3), CD244 (SLAMF4), CD84 (SLAMF5), CD352 (SLAMF6), CD319 (SLAMF7), CD353 (SLAMF8) and CD84-H1 (SLAMF9) (14). These are surface molecules that are expressed on hematopoietic cells. They are also a type of immune molecule that can regulate innate and adaptive immunity by activating associated immune cells $(14,15)$. Among these nine molecules, SLAMF6 (Ly108 in mice) is highly expressed in activated T and B cells. Its expression can also be increased in dendritic cells and macrophages via inflammatory signals (16), functioning as both a positive and negative regulator of the immune response (17). SLAMF6 can inhibit the secretion of T-dependent and T-independent antibodies (18). It has also been reported that SLAMF6 can activate natural killer cells to induce cytotoxicity and influence IFN- $\gamma$ production $(19,20)$. SLAMF3 is involved in the inhibition of HCC progression and cell proliferation $(21,22)$. In addition to SLAMF6, there are several SLAMF family members that are expressed on the macrophage surface, including SLAMF3, SLAMF4, SLAMF5 and SLAMF7 (23). SLAMFs have been demonstrated to negatively influence the function of macrophages by reducing IL-6 production and increasing IL-13 secretion (24).

As a membrane protein that is expressed on immune cells, SLAMF6 has been proven to serve a role in numerous diseases (25-28). For example, although the expression of SLAMF6 on peripheral $\mathrm{T}$ cells is not markedly different in patients with systemic lupus erythematosus, it has been determined that the co-stimulatory function of SLAMF6 is lacking (25). In addition, SLAMF6 can promote Th17 differentiation (26). SLAMF6 can also stimulate the interaction between colonic innate immune cells and Gram-negative bacteria, thus reducing mucosal protection and enhancing inflammation, resulting in lethal colitis in mice (27). The administration of anti-SLAMF6 antibodies combined with ibrutinib, which is a Bruton tyrosine kinase inhibitor that serves as an anticancer targeted drug, can effectively eliminate tumor cells in the spleen, bone marrow, liver and peritoneal cavity (28). SLAMF6 can affect the function of Th2 by binding to downstream proteins and transcription factors, such as nuclear factor- $\kappa B(N F-\kappa B)(29)$, which serves a role in macrophage polarization (30).
The present study investigated the expression of SLAMF6 in the peripheral $\mathrm{CD} 14^{+}$monocytes of patients with HCC to determine whether this expression was associated with the clinicopathological characteristics of these patients. Furthermore, SLAMF6 expression on different phenotype macrophages was detected in order to explore the relationship between this and HCC progression. This may provide a novel therapeutic target for HCC treatment.

\section{Materials and methods}

Blood sampling and mononuclear cell isolation. Blood samples were obtained from 34 patients with HCC and 33 healthy individuals at the Shandong Cancer Hospital and Institute (Jinan, China). Patients with HCC did not receive any form of antitumor therapy and were pathologically diagnosed with HCC prior to sampling. None of the healthy donors were positive for hepatitis B virus (HBV), hepatitis C virus or human immunodeficiency virus, consumed excessive alcohol or received any chemotherapy prior to sampling. Human peripheral mononuclear cell isolation was performed by centrifuging $\left(800 \mathrm{x} \mathrm{g} ; 15 \mathrm{~min} ; 25^{\circ} \mathrm{C}\right)$ blood samples with an $\mathrm{EZ}_{-\mathrm{Sep}^{\mathrm{TM}}}$ lymphocyte separation tube (Dakewe Biotech Co., Ltd.). The mono-macrophages were marked with corresponding markers immediately after the isolation. The present study was approved by the Medical Ethics Committee of Shandong Cancer Hospital and Institute and informed consent was obtained from each participant. The clinicopathological characteristics of the healthy donors and patients with HCC are summarized in Table I.

Mouse HCC model establishment and macrophage isolation. C57BL/6 mice were purchased from Beijing Weitonglihua Laboratory Animal Co., Ltd. and maintained in a controlled room (specific pathogen free; 12-h light/dark cycle; free access to food and water, temperature, $20-26^{\circ} \mathrm{C}$; relative humidity, 40-70\%). All experiments involving mice were approved by the Animal Care Committee of Shandong Cancer Hospital and Institute and performed according to the Animal Management Rules of the Chinese Ministry of Health. To establish a murine model of HCC, $100 \mu 1$ PBS solution containing $1 \times 10^{6} \mathrm{H} 22$ cells (cat. no. CL-0341; American Type Culture Collection) was subcutaneously injected into eight C57BL/6 male mice (8-10 weeks old; average weight, $22.32 \pm 0.19 \mathrm{~g}$ ). After 14 days, the mice were euthanized via intraperitoneal injection of $100 \mathrm{mg} / \mathrm{kg}$ sodium pentobarbital followed by cervical dislocation (average weight, $24.17 \pm 0.62 \mathrm{~g}$ ). The subsequent experiments were conducted immediately after the tissues were collected. The infiltrating lymphocytes from each type of tissue were isolated by centrifugation $(800 \mathrm{x} \mathrm{g} ; 20 \mathrm{~min}$; $25^{\circ} \mathrm{C}$ ) over $40 \%$ Percoll solution (GE Healthcare) (31), after which the expression level of SLAMF6 was evaluated by flow cytometry.

Flow cytometry. Flow cytometry was performed to detect the expression of SLAMF6 and various surface markers in macrophages. Human peripheral blood mononuclear cells (CD14 ${ }^{+}$ were stained with anti-human SLAMF6-PE [cat. no. 317208; $3 \mu 1$ stock solution/test $\left(1 \times 10^{6}\right.$ cells $)$; BioLegend, Inc.] and anti-human CD14-FITC [cat. no. 301804; $5 \mu$ l stock 
Table I. Clinicopathological characteristics of patients with hepatocellular carcinoma and healthy donors.

\begin{tabular}{lrc}
\hline Variable & $\begin{array}{c}\text { HCC patients } \\
(\mathrm{n}=34)\end{array}$ & $\begin{array}{c}\text { Healthy donors } \\
(\mathrm{n}=33)\end{array}$ \\
\hline Sex & & \\
Male, $\mathrm{n}(\%)$ & $26(76.47)$ & $25(75.76)$ \\
Female, $\mathrm{n}(\%)$ & $8(23.53)$ & $8(24.24)$ \\
Age, mean years (range) & $60(41-80)$ & $44(21-62)$ \\
HBV-DNA, $\mathrm{n}(+, \%)$ & $12(35.29)$ & 0 \\
AFP, n $(>100, \%)$ & $14(41.18)$ & 0 \\
Single tumor, $\mathrm{n}(\%)$ & $13(38.24)$ & 0 \\
Lymphatic metastasis, $\mathrm{n}(\%)$ & $10(29.41)$ & 0 \\
Distant metastasis, $\mathrm{n}(\%)$ & $7(20.59)$ & 0 \\
TNM stage I-II, $\mathrm{n}(\%)$ & $13(38.24)$ & 0 \\
\hline
\end{tabular}

AFP, $\alpha$-fetoprotein; HBV, hepatitis B virus; TNM, Tumor-NodeMetastasis.

solution/test $\left(1 \times 10^{6}\right.$ cells); BioLegend, Inc.] antibodies for $30 \mathrm{~min}$ at $4^{\circ} \mathrm{C}$. Furthermore, murine macrophages $\left(\mathrm{CD} 11 \mathrm{~b}^{+}\right)$ were stained with anti-mouse SLAMF6-PE [cat. no. 2124557; $3 \mu \mathrm{l}$ stock solution/test $\left(1 \times 10^{6}\right.$ cells); eBioscience; Thermo Fisher Scientific, Inc.] and anti-mouse CD11b-PE-cy 7 [cat. no. E07514-1633; $2 \mu 1$ stock solution/test ( $1 \times 10^{6}$ cells); eBioscience; Thermo Fisher Scientific, Inc.] antibodies for $30 \mathrm{~min}$. A total of $\sim 10,000$ cells were analyzed using BD FACSAria II (BD Biosciences) equipment and cells were gated according to their forward and side scatter properties.

Cell culture. Murine HCC cell lines, including H22 [cultured in RMPI-1640 containing 10\% FBS and 1\% penicillin/streptomycin mixture (cat. no. 15140-122; Gibco; Thermo Fisher Scientific, Inc.)] and Hepa1-6 (cat. no. CL-0105; cultured DMEM containing $10 \%$ FBS and $1 \%$ penicillin/streptomycin mixture), were obtained from the American Type Culture Collection. Murine peripheral macrophages (PMs) and bone marrow-derived macrophages (BMDMs) were cultured in DMEM containing $10 \%$ FBS and $1 \%$ penicillin/streptomycin mixture and placed at $37^{\circ} \mathrm{C}$ in a humidified incubator containing $5 \% \mathrm{CO}_{2}$. The human monocyte cell line THP-1 (cat. no. BNCC100407; BeNa Culture Collection; Beijing Beina Chunglian Institute of Biotechnology) was cultured in RMPI-1640 supplemented with $10 \%$ FBS and $1 \%$ penicillin/streptomycin mixture. DMEM (cat. no. C11995500CP) was obtained from Gibco; Thermo Fisher Scientific, Inc., RMPI-1640 (cat. no. SH30809.01) was purchased from HyClone; Cytiva and FBS (cat. no. WXBD5226V) was obtained from Sigma-Aldrich; Merck KGaA.

Macrophage polarization. Mouse peritonitis was induced by intraperitoneal injection of $1 \mathrm{ml}$ sterile $6 \%$ starch solution in six 8-10 week old male C57BL/6 mice. After $72 \mathrm{~h}$, mice were euthanized via intraperitoneal injection of $100 \mathrm{mg} / \mathrm{kg}$ sodium pentobarbital followed by cervical dislocation. The peritoneal cells were harvested by irrigating the abdominal cavity and the PMs were enriched by quick adhesion. Model establishment, harvesting of cells and quick adhesion were described previously (32). PMs were polarized into M1 phenotypes using $100 \mathrm{ng} / \mathrm{ml}$ lipopolysaccharide (LPS) for $24 \mathrm{~h}$ at $37^{\circ} \mathrm{C}$, while M2 macrophages were polarized using $20 \mathrm{ng} / \mathrm{ml} \mathrm{IL-4}$ for $24 \mathrm{~h}$ at $37^{\circ} \mathrm{C}$. PBS was used as a control. Furthermore, six 4-6 week old male C57BL/6 mice were euthanized via intraperitoneal injection of $100 \mathrm{mg} / \mathrm{kg}$ sodium pentobarbital followed by cervical dislocation. The BMDMs were collected by flushing the femurs and tibias (32). BMDMs were polarized into the M1 phenotype using $20 \mathrm{ng} / \mathrm{ml}$ granulocyte macrophage colony-stimulating factor (cat. no. AF-315-03-20; PeproTech, Inc.) treatment for 5 days at $37^{\circ} \mathrm{C}$, while the $\mathrm{M} 2$ phenotype was obtained using $100 \mathrm{ng} / \mathrm{ml}$ macrophage colony-stimulating factor (cat. no. AF-315-02-10; PeproTech, Inc.) treatment for 5 days at $37^{\circ} \mathrm{C}$. Samples were then stimulated using $100 \mathrm{ng} / \mathrm{ml}$ LPS for $24 \mathrm{~h}$ to induce M1 or $20 \mathrm{ng} / \mathrm{ml} \mathrm{IL-4}$ for $24 \mathrm{~h}$ to induce M2.

HCC conditioned medium (HCM). After culturing $\mathrm{H} 22$ cells for $24 \mathrm{~h}$, serum-free DMEM was collected for use as HCM. Newly isolated PMs $\left(2 \times 10^{5}\right.$ per well) seeded into 12-well-plates were exposed to $200 \mu \mathrm{l} \mathrm{HCM}$, producing a final volume of $1,000 \mu \mathrm{l}$ with complete medium (DMEM containing $10 \%$ FBS). The control group was treated with $200 \mu \mathrm{l}$ FBS-free DMEM added to $800 \mu \mathrm{l}$ complete medium. The final percentage of HCM was $20 \%$.

SLAMF6 interference. SLAMF6 expression was inhibited in murine PMs via transfection with SLAMF6 small interfering RNA (siRNA). Three siRNAs were tested and all sequences were working. The sequences of the siRNAs are presented in Table II. SLAMF6 siRNA (120 pmol) or non-targeting negative control siRNA (120 pmol) (Biosune Biotechnology Co., Ltd.) were transfected into the PMs in each of the 12 wells using lipofectamine RNAiMAX (cat. no. 13778-150; Invitrogen; Thermo Fisher Scientific, Inc.) for $24 \mathrm{~h}$ at $37^{\circ} \mathrm{C}$. After $24 \mathrm{~h}$, the PMs, which had been transfected with siRNAs, were treated with Bay11-7082 (10 $\mu \mathrm{M}$; cat. no. HY-13453; MedChemExpress), an inhibitor of NF- $\mathrm{B}$, for $2 \mathrm{~h}$ before IL-4 (20 ng/ml) was added for $24 \mathrm{~h}$. SLAMF6-silenced PMs, negative control PMs and their culture supernatants were collected (immediately or after storage at $-80^{\circ} \mathrm{C}$ in an ultra-low temperature freezer within 1 month) to examine the effect of SLAMF6 on cell proliferation and invasion, since macrophages exert their function on tumor cells via secretion of cytokines.

Analysis of tumor cell migration, invasion and proliferation, and in vivo assessment. The culture supernatant of SLAMF6-silenced PMs and control PMs was used to culture Hepa1-6 cells. Transwell assay cell migratory and invasive abilities were determined using 24-well transwell chambers with an $8-\mu \mathrm{m}$ pore polycarbonate membrane insert (Corning, Inc.; cat. no. 3422). For invasion assay only, the membrane was pre-coated with $50 \mu 1$ Matrigel (cat. no. 356234; BD Biosciences) for half an hour. Then, $\sim 2.5 \times 10^{4}$ Hepa1- 6 cells suspended in $200 \mu \mathrm{l}$ serum-free DMEM were loaded onto the upper chamber, while $600 \mu \mathrm{l}$ medium collected from corresponding macrophages, as aforementioned in the SLAMF6 interference section, was added to the lower chamber. After $16 \mathrm{~h}$ (migration) or $24 \mathrm{~h}$ (invasion), the cells on the lower 
Table II. Sequence of Ly108 small interfering RNAs used for transfection and of primers used for RT-qPCR.

\begin{tabular}{|c|c|}
\hline Primer & Sequence \\
\hline \multicolumn{2}{|l|}{ Small interfering RNA } \\
\hline Control, sense & UUCUCCGAACGUGUCACGUTT \\
\hline Ly108-366, sense & GCUAAUGAAUGGCGUUCUATTUAGAACGCCAUUCAUUAGCTT \\
\hline Ly108-567, sense & CCUGCAAAUCAGCAACCUUTTAAGGUUGCUGAUUUGCAGGTT \\
\hline Ly108-1190, sense & CCAUGACAAUUUACUCCAUTTAUGGAGUAAAUUGUCAUGGTT \\
\hline \multicolumn{2}{|l|}{ RT-qPCR } \\
\hline Homo- $\beta$-actin, forward & CATGTACGTTGCTATCCAGGC \\
\hline Homo- $\beta$-actin, reverse & CTCCTTAATGTCACGCACGAT \\
\hline Homo-SLAMF6, forward & GGCCCAGGGAATGTAGTTTCA \\
\hline Homo-SLAMF6, reverse & ACTGACTCCCCCAGAATCCC \\
\hline Homo-TNF- $\alpha$, forward & GAGGCCAAGCCCTGGTATG \\
\hline Homo-TNF- $\alpha$, reverse & CGGGCCGATTGATCTCAGC \\
\hline Mouse- $\beta$-actin, forward & CGGGCCGATTGATCTCAGC \\
\hline Mouse- $\beta$-actin, reverse & TACAGCCCGGGGAGCATCGT \\
\hline Mouse-ly 108, forward & CCTTCAGGGTAATGGGTTGGTT \\
\hline Mouse-ly 108, reverse & CCTTCAGGGTAATGGGTTGGTT \\
\hline Mouse-arg-1, forward & TGTCCCTAATGACAGCTCCTT \\
\hline Mouse-arg-1, reverse & GCATCCACCCAAATGACACAT \\
\hline Mouse-TNF- $\alpha$, forward & GCCACCACGCTCTTCTGTCT \\
\hline Mouse-TNF- $\alpha$, reverse & TGAGGGTCTGGGCCATAGAAC \\
\hline Mouse-iNOS, forward & GCCACCAACAATGGCAACAT \\
\hline Mouse-iNOS, reverse & TCGATGCACAACTGGGTGAA \\
\hline Mouse-IL-1 $\beta$, forward & ACCTTCCAGGATGAGGACATGA \\
\hline Mouse-IL-1 $\beta$, reverse & AACGTCACACACCAGCAGGTTA \\
\hline
\end{tabular}

Arg-1, arginase-1; IL-1 $\beta$, interleukin $1 \beta$; iNOS, inducible nitric oxide synthase; RT-qPCR, reverse transcription quantitative PCR; SLAMF6, signaling lymphocytes activation molecule family member 6; TNF- $\alpha$, tumor necrosis factor- $\alpha$.

chamber were stained with $0.1 \%$ crystal violet for $10 \mathrm{~min}$ at room temperature. The number of invaded cells were counted under a light microscope (magnification, x200).

To prepare murine hepatoma homografts, $2 \times 10^{5} \mathrm{H} 22$ cells in $100 \mu \mathrm{l}$ PBS were subcutaneously injected into the groins of 14 C57BL/6 male mice (8-10 week old; average weight, $22.72 \pm 0.17 \mathrm{~g}$ ), which were anesthetized by intraperitoneal injection with $50 \mathrm{mg} / \mathrm{kg}$ sodium pentobarbital. At 8 days after H22 cell injection, SLAMF6-silenced PMs and control PMs were injected into the tumor site, after which time the size of tumors was measured. Tumor volume was calculated using the following formula: $1 / 2 \mathrm{ab}^{2}$ ( $a$, long diameter; $b$, short diameter). The procedure was repeated every other day. On the 18th day, all mice were euthanized via intraperitoneal injection of $100 \mathrm{mg} / \mathrm{kg}$ sodium pentobarbital followed by cervical dislocation (average weight, 25.03 $\pm 0.71 \mathrm{~g}$ ), after which tumors were collected and their size and weight were determined. The maximum tumor volume was as follows: $1 / 2 \times 17.0 \times 16.5 \times 16.5=2,314.125 \mathrm{~mm}^{3}$.

$R N A$ isolation and reverse transcription quantitative (RT-q) $P C R$. According to the manufacturer's protocol, RNA was extracted from THP-1 cells and PMs using the Fastagen RNA isolation kit (cat. no. 220011; Fastagen Biotech).
Subsequently, RNA was reverse transcribed into cDNA using the Takara reverse transcription kit (cat. no. RR037A; Takara Biotechnology Co., Ltd.). The PCR thermocycling conditions (Bio-Rad Laboratories, Inc.) were as follows: $95^{\circ} \mathrm{C}$ for $30 \mathrm{sec}$, followed by 40 cycles of $95^{\circ} \mathrm{C}$ for $5 \mathrm{sec}$. The relative expression levels were normalized to endogenous control and were expressed as $2^{-\Delta \Delta C q}(33)$. The primers used for PCR are listed in Table II.

Western blotting. PMs were transfected with negative control or Ly108 siRNA and lysed with RIPA lysis buffer (cat. no. P0013B; Beyotime Institute of Biotechnology) at $4^{\circ} \mathrm{C}$. Proteins $(5 \mu \mathrm{g} /$ lane $)$, the concentration of which was determined by the BCA protein determination method (cat.no. A53225; Thermo Fisher Scientific, Inc.), were separated by $10 \%$ SDS-PAGE and transferred onto PVDF membranes. After blocking membranes with $5 \%$ bovine serum albumin (cat. no. A8010; Beijing Solarbio Science \& Technology Co., Ltd.) for $2 \mathrm{~h}$ at room temperature, membranes were incubated with primary antibodies against phosphorylated (p)-NF- $\kappa \mathrm{B}$ p65 (Cell Signaling Technology, Inc.; cat. no. 3033; 1:1,000); NF- $\mathrm{B}$ (Cell Signaling Technology, Inc.; cat. no. 8242; 1:1,000) and $\beta$-actin (Cell Signaling Technology, Inc.; cat. no. 3700; $1: 1,000)$ at $4^{\circ} \mathrm{C}$ overnight. The secondary antibodies 
(anti-mouse IgG, HRP-linked, cat. no. 7076S, 1:2,000, Cell Signaling Technology, Inc.; anti-rabbit $\operatorname{IgG}(\mathrm{H}+\mathrm{L})$ antibody conjugated to biotin, cat. no. 14708S, 1:2,000, Cell Signaling Technology, Inc.) were incubated with the membranes at $25^{\circ} \mathrm{C}$ for $1 \mathrm{~h}$. A western blot Imaging System (Tanon Science and Technology Co., Ltd.) was used to detect protein bands and obtain images using the Alldoc-ECL 2019 software (Tanon Science and Technology Co., Ltd.). The data were analyzed via densitometry and normalized to expression of the internal control ( $\beta$-actin).

Statistical analysis. All data were analyzed using GraphPad Prism 8 software (GraphPad Software Inc.). A Student's t-test was used to determine differences between two groups. One-way ANOVA followed the Tukey's post hoc test was used to determine the differences between more than two groups. Data are presented as the mean \pm standard error of the mean. $\mathrm{P}<0.05$ was considered to indicate a statistically significant difference.

\section{Results}

Enhanced SLAMF6 expression in TAMs is related to the severity of HCC. SLAMF6 expression level was detected in the peripheral $\mathrm{CD} 14^{+}$monocytes of patients with $\mathrm{HCC}$ and healthy donors. As presented in Fig. 1A, the percentage of peripheral $\mathrm{CD}^{+} 4^{+}$monocytes expressing SLAMF6 in patients with HCC was significantly higher compared with healthy donors. The relationship between SLAMF6 expression level and the severity of HCC was subsequently analyzed. As presented in Fig. 1B, the expression level of SLAMF6 was assessed in six groups of patients categorized according to the following criteria: i) The HBV status (negative or positive); ii) the $\alpha$-fetoprotein (AFP) level ( $>$ or $<100 \mathrm{ng} / \mathrm{ml}$ ); iii) the number of tumors (single or multiple); iv) the lymphatic metastasis occurrence (yes or no); v) the distant metastasis occurrence (yes or no); and vi) the different stages of tumors. Among these groups, only two presented with differences in SLAMF6 expression. The expression level of SLAMF6 in $\mathrm{CD}_{1} 4^{+}$monocytes from patients with $\mathrm{HCC}$ who were HBV-positive was significantly higher than in HBV-negative individuals. Furthermore, an increased expression of SLAMF6 was demonstrated in patients with HCC whose serum AFP level was $>100 \mathrm{ng} / \mathrm{ml}$ compared with those exhibiting AFP level $<100 \mathrm{ng} / \mathrm{ml}$, which indicated a relationship between SLAMF6 expression and HCC poor prognosis (34). Since the age distribution of the patients and the healthy donors was too different for SLAMF6 expression, we made the comparison between the low and high age groups and found no difference between the two age groups (Fig. S1).

To determine the expression level of SLAMF6 in TAMs, a murine tumor model of HCC was established, after which the expression of Ly108 was determined in TAMs isolated from tumor and liver tissues. As presented in Fig. 1C, when compared with liver tissues, the TAMs isolated from tumor tissues expressed a higher level of Ly108. These results demonstrated that SLAMF6 expression was increased in TAMs, which may be associated with the poor prognosis of patients with HCC.

M2-like macrophages exhibit a higher SLAMF6 expression. As previously reported, TAMs are considered to be M2-like macrophages (35). The present study therefore compared the expression level of SLAMF6 in different types of macrophages. To achieve this, human THP-1, murine PMs and murine BMDMs were polarized into M1- and M2-like macrophages. As presented in Fig. 2A, different expression levels of TNF- $\alpha$, a M1 marker, indicated that polarization was successful. In addition, the results from RT-qPCR demonstrated that the human M2-like phenotype exhibited higher SLAMF6 expression level compared with the M1-like phenotype. However, the results from flow cytometry analysis revealed that the human M1-like phenotype expressed a higher percentage of SLAMF6 compared with the M2-like phenotype.

For murine PMs, the mean fluorescence intensity (MFI) of Ly108 expression in the M2-like phenotype was higher than that in the M1-like phenotype. However, the percentage expression of Ly108 did not significantly differ between the two groups according to flow cytometry analysis (Fig. 2B), which may be due to the fact that the PMs we collected were induced by the starch and already expressed $\sim 90 \%$ Ly108. Regarding murine BMDMs, the percentage of expression and MFI of Ly108 in M2-like macrophages were higher than in M1-like macrophages (Fig. 2C). These results suggested that the M2-like phenotype exhibited higher SLAMF6 expression level compared with the M1-like phenotype.

Macrophage SLAMF6 expression and polarization is promoted in the tumor microenvironment. The aforementioned results indicated that the M2-like phenotype exhibited increased SLAMF6 expression level. Thus, the present study aimed to determine the reason behind this increase. The effect of HCC microenvironment on PMs was therefore assessed by using HCM. As presented in Fig. 3A, $200 \mu \mathrm{l}$ HCM significantly promoted Ly108 expression in PMs and induced two positive peaks rather than one positive peak following the negative peak. This result may be due to the fact that HCM enhanced Ly108 expression in macrophages that already expressed a certain amount of Ly108, and HCM might trigger the Ly108 expression in macrophages that did express the molecule at a lower level. In addition, the expression levels of M2 marker arginase-1 (arg-1) and of the M1 markers TNF- $\alpha$, IL- $1 \beta$ and inducible nitric oxide synthase (iNOS) were increased in the HCM group compared with the control group (Fig. 3B). It was therefore hypothesized that the tumor microenvironment may enhance SLAMF6 expression and that an unknown mechanism may promote M2 polarization in these conditions.

Ly108 silencing reverses M2 polarization and inhibits HCC progression. Previous studies have revealed that M2-like macrophages can promote HCC growth and invasion $(6,8,12)$. Ly108 siRNA was therefore used to investigate the role of Ly108 in macrophage polarization and HCC progression. As presented in Fig. 4A, Ly108 expression was successfully decreased following siRNA transfection. The results also demonstrated that following Ly108 silencing, the expression of the M2 marker arg- 1 was decreased, while the expression of certain M1 markers, including TNF- $\alpha$, IL-1 $\beta$ and iNOS, was increased. The culture supernatant of macrophages treated with Ly108 or control siRNA was subsequently co-cultured with Hepa1-6 cells (Fig. 4B). Following co-culture, the proliferation, 
A

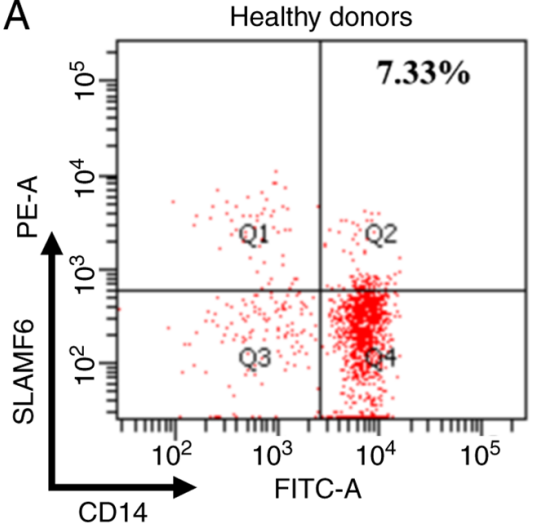

$\mathrm{B}$
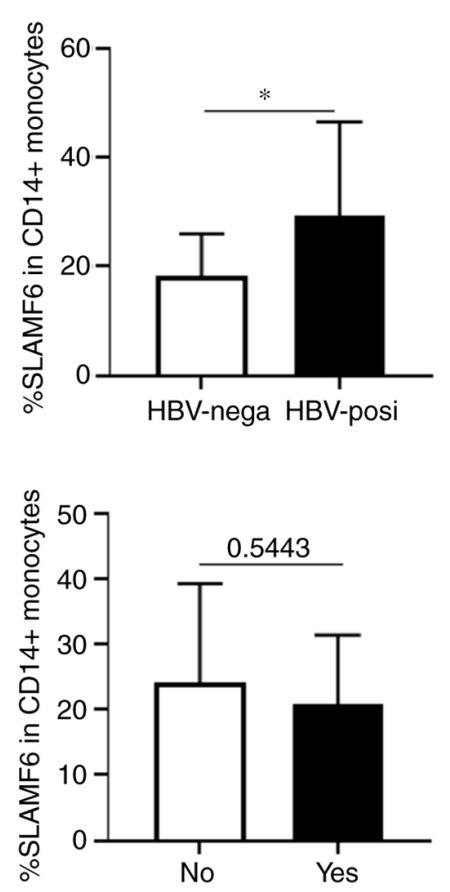

(Lymphatic metastasis)

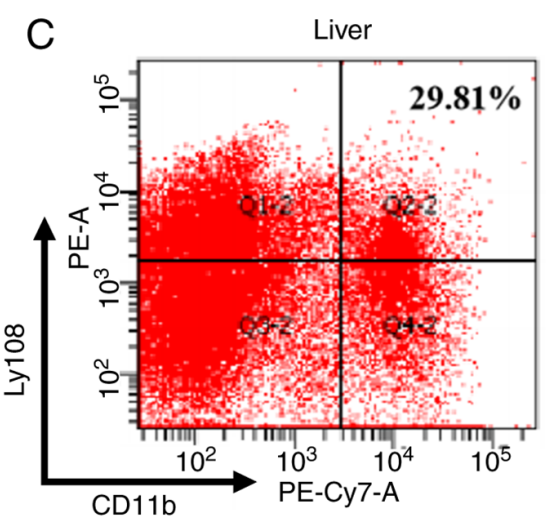

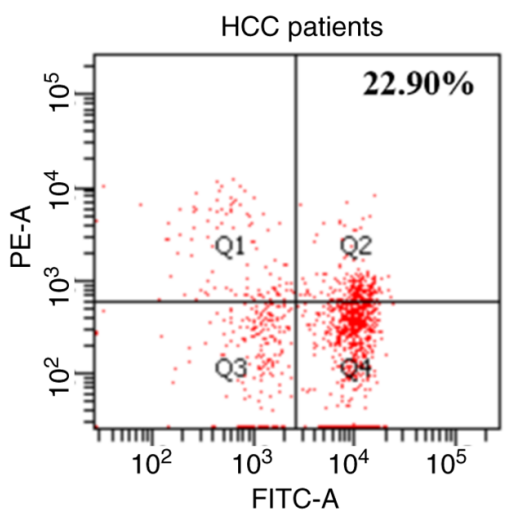
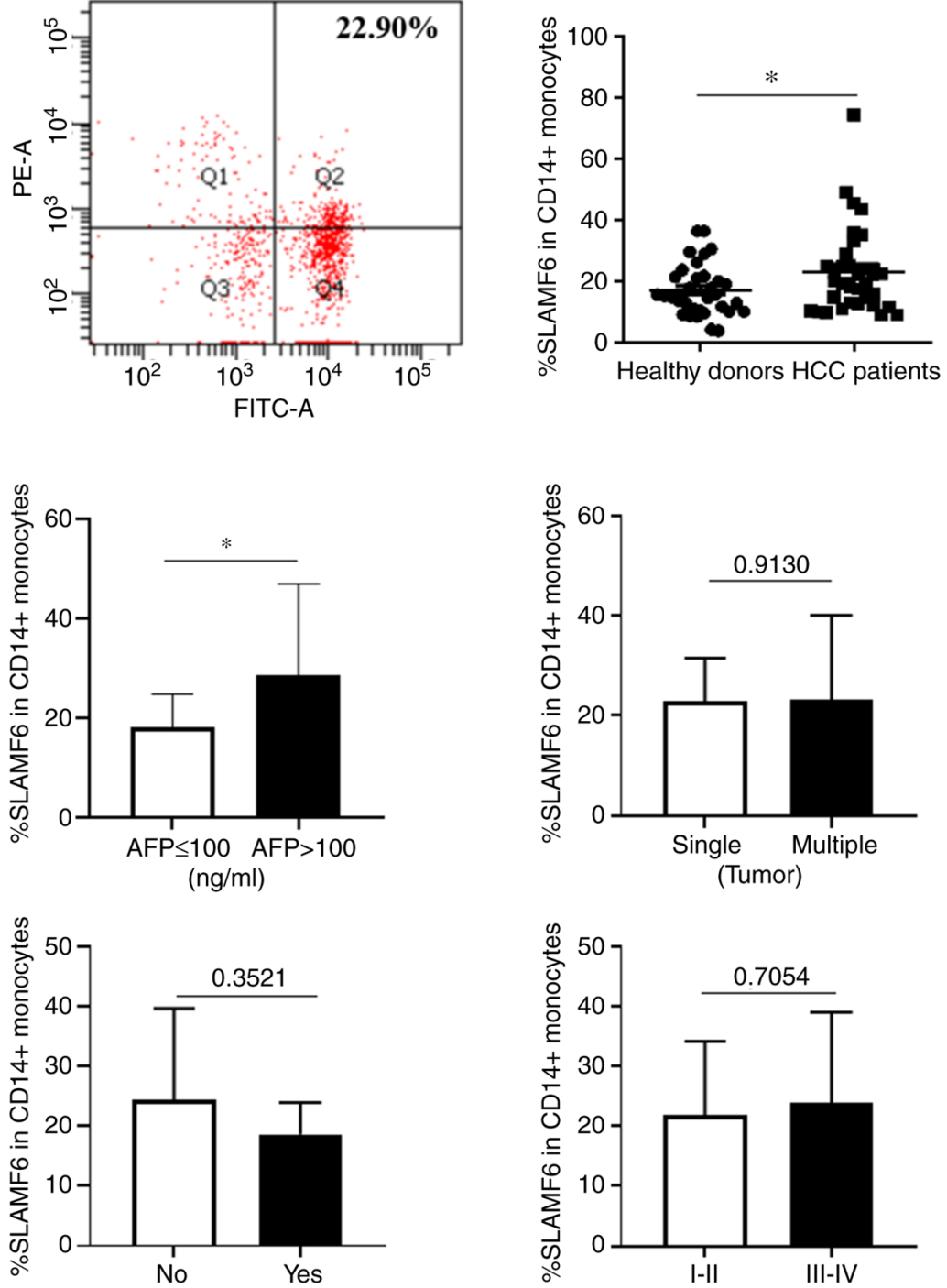

(Distant metastasis)

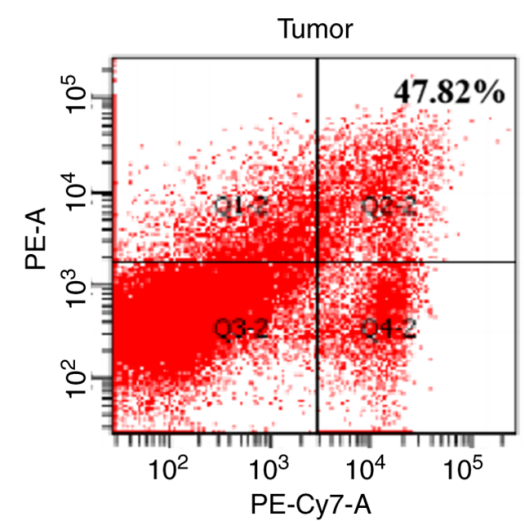

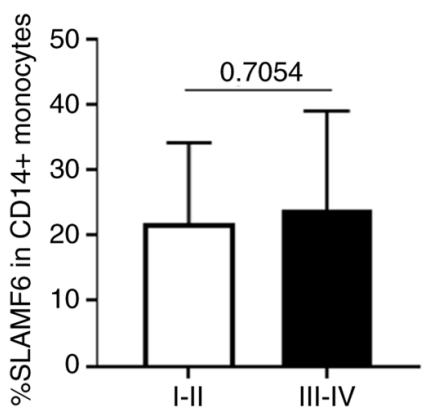

(TNM staging)

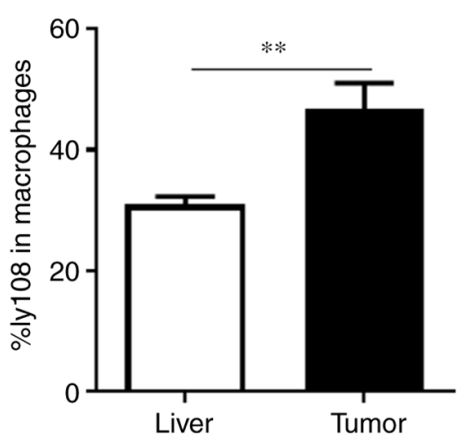

Figure 1. SLAMF6 expression level is increased in monocytes and tumor-associated macrophages. Peripheral CD14 ${ }^{+}$monocytes were isolated from 34 patients with HCC and 33 healthy donors. (A) Flow cytometry analysis was used to measure the percentage of SLAMF6 ${ }^{+}$CD14 ${ }^{+}$cells among monocytes. The monocytes were assessed using $\mathrm{CD}_{14}{ }^{+}$in human peripheral blood mononuclear cells. (B) Association between SLAMF6 expression level and the clinicopathological characteristics of patients with HCC. (C) Using flow cytometry, Ly108 expression level was evaluated in the infiltrating macrophages (assessed using

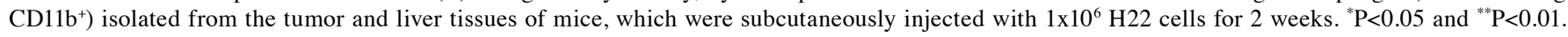
SLAMF6, signaling lymphocyte activation molecule family member 6; HCC, hepatocellular carcinoma.

migration and invasion of Hepa1-6 cells was significantly decreased. These results suggested that Ly108 could inhibit the migratory and invasive abilities of Hepa1-6 cells.
Macrophage Lyl08 promotes HCC growth in vivo. To improve our understanding of the effect of TAM Ly108 on HCC progression in vivo, a murine model of HCC was 
A

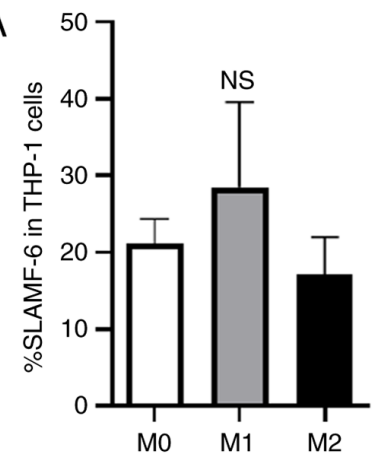

B

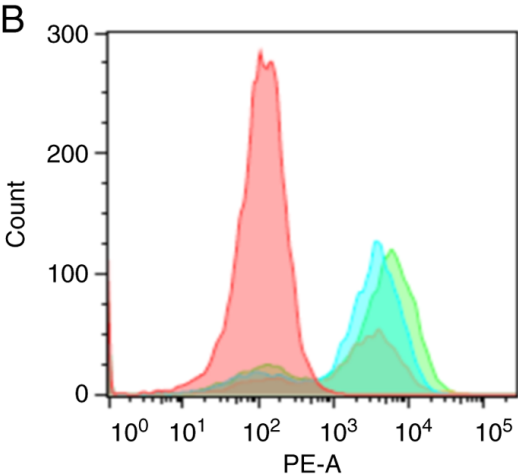

\begin{tabular}{|l|lr|}
\hline & \multicolumn{2}{|l|}{ Mean: PE-A } \\
\hline & Isotype & 137 \\
\hline & M0 & 4030 \\
\hline & M1 & 3964 \\
\hline & M2 & 5470 \\
\hline
\end{tabular}

\section{C}

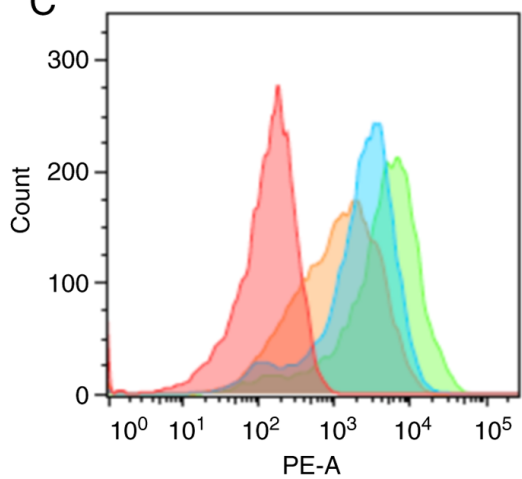

\begin{tabular}{|l|lr|}
\hline & \multicolumn{2}{|l|}{ Mean: PE-A } \\
\hline & Isotype & 169 \\
\hline$\square$ & M0 & 3352 \\
\hline$\square$ & M1 & 1919 \\
\hline$\square$ & M2 & 6643 \\
\hline
\end{tabular}
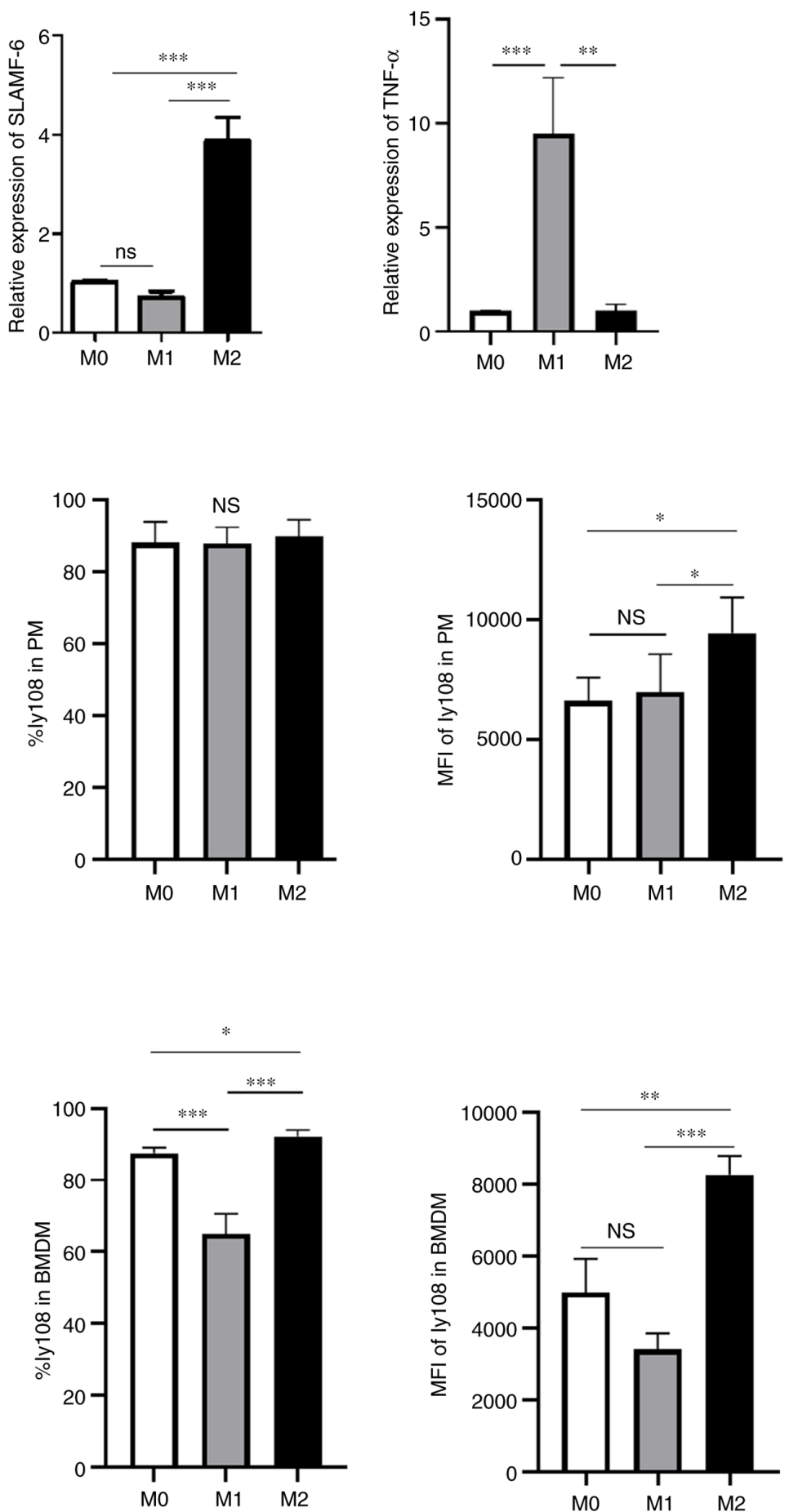

Figure 2. M2-like macrophages exhibit higher SLAMF6 expression level. (A) Flow cytometry analysis was used to determine the percentage of SLAMF6 THP-1 cells, and the relative expression of SLAMF6 and TNF- $\alpha$ was evaluated via reverse transcription quantitative PCR. The percentage of Ly108 ${ }^{+}$cells, along with the mean fluorescence intensity of Ly108 expression was also determined in (B) PMs and (C) BMDMs. ${ }^{*}<<0.05,{ }^{* *} \mathrm{P}<0.01$ and ${ }^{* * *} \mathrm{P}<0.001 . \mathrm{NS}$, no significance; SLAMF6, signaling lymphocyte activation molecule family member 6; PM, peripheral macrophage; BMDM, bone marrow-derived macrophage; TNF- $\alpha$, tumor necrosis factor $\alpha$.

established. Ly108 siRNA or control siRNA was transfected into M2-like macrophages, which were subsequently injected into C57BL/6 mice. The results demonstrated that, compared with control M2-like macrophages, Ly108 siRNA-transfected M2-like macrophages inhibited the growth of H22 tumors in terms of growth rate, gross tumor volume and tumor weight (Fig. 5A).

Ly108 promotes $H C C$ growth via the $N F-\kappa B$ pathway. The present study aimed to determine the potential underlying 
A

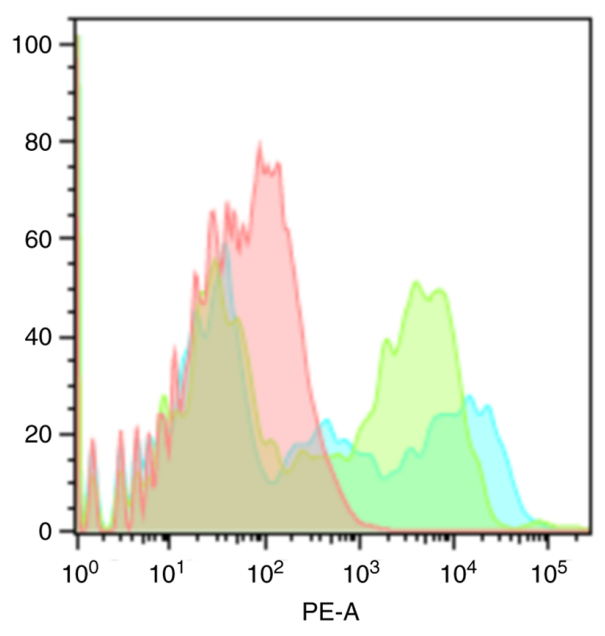

\begin{tabular}{|l|ll|}
\hline & \multicolumn{2}{|c|}{ Mean: PE-A } \\
\hline & Isotype & 88.4 \\
\hline$\square$ & HCM-0 & 2864 \\
\hline$\square$ & HCM-200 & 3427 \\
\hline
\end{tabular}

B
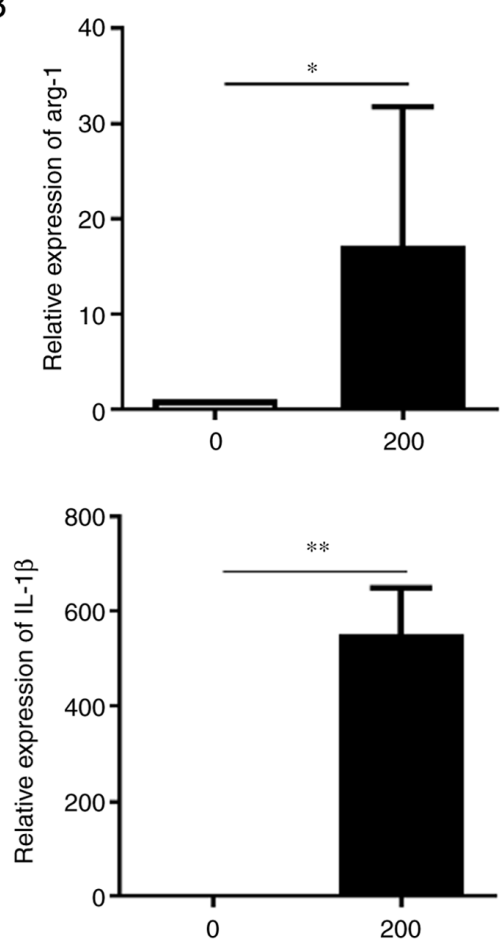
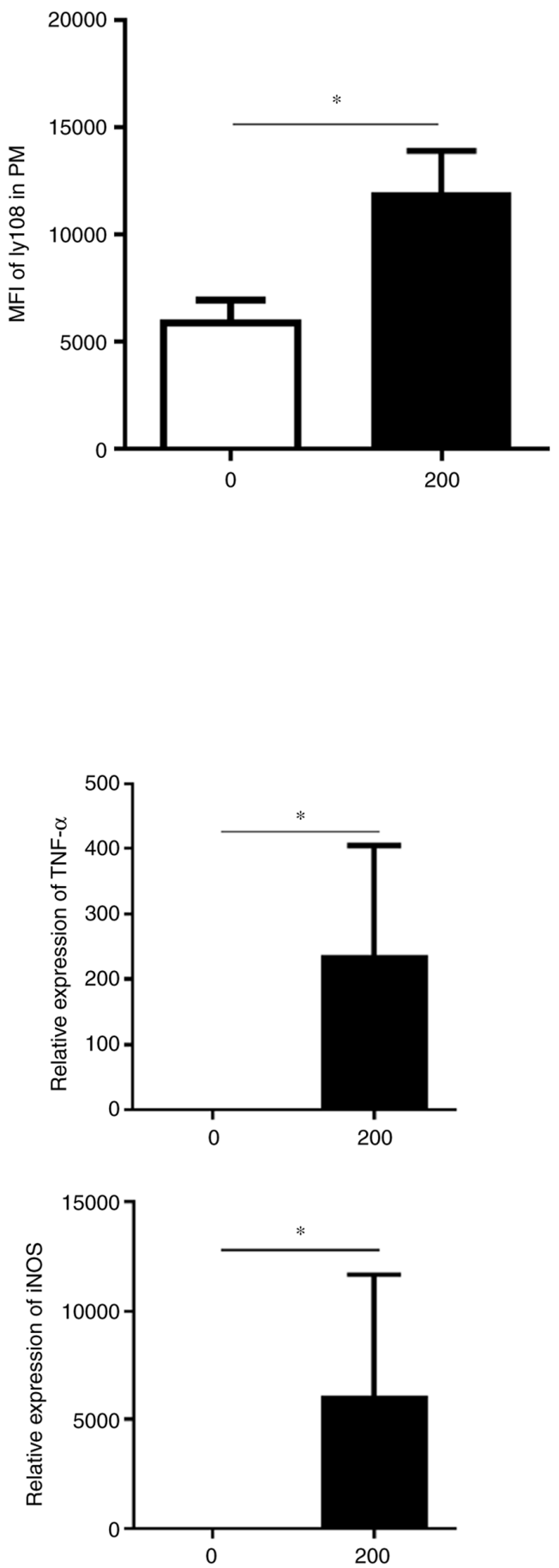

Figure 3. Ly108 expression and macrophage polarization is promoted by the tumor microenvironment. (A) Flow cytometry was performed to analyze the expression of Ly108, after which the mean fluorescence intensity of Ly108 was measured in PMs cultured with or without hepatocellular carcinoma conditioned medium. (B) Reverse transcription quantitative PCR was subsequently performed to determine the relative expression of arg- $1, \mathrm{TNF}-\alpha$, IL-1 $\beta$ and iNOS in PMs. " $\mathrm{P}<0.05$ and ${ }^{* *} \mathrm{P}<0.01$. PM, peritoneal macrophage; arg-1, arginase-1; IL-1 $\beta$, interleukin $1 \beta$; iNOS, inducible nitric oxide synthase; TNF- $\alpha$, tumor necrosis factor- $\alpha$.

mechanism of Ly108. As presented in Fig. 5B, the Ly108 siRNA-transfected group presented higher expression of p65 phosphorylation. Furthermore, treatment with Bay11-7082, an inhibitor of NF- $\kappa \mathrm{B}$, inhibited the phosphorylation of p65. In addition, when Ly108 siRNA-transfected macrophages were treated with Bay11-7082, they were able to re-polarize to the M2 phenotype. This result was determined according to higher Arg- 1 and IL- $1 \beta$ levels and lower TNF- $\alpha$ and iNOS levels (Fig. 5C). Macrophages of which phenotype was reversed by Bay11-7082 treatment 
A

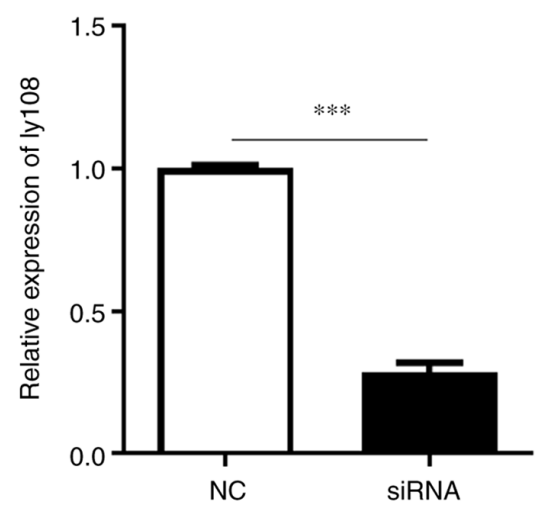

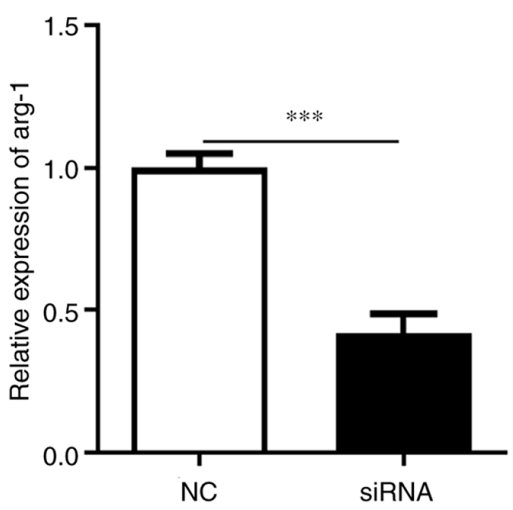
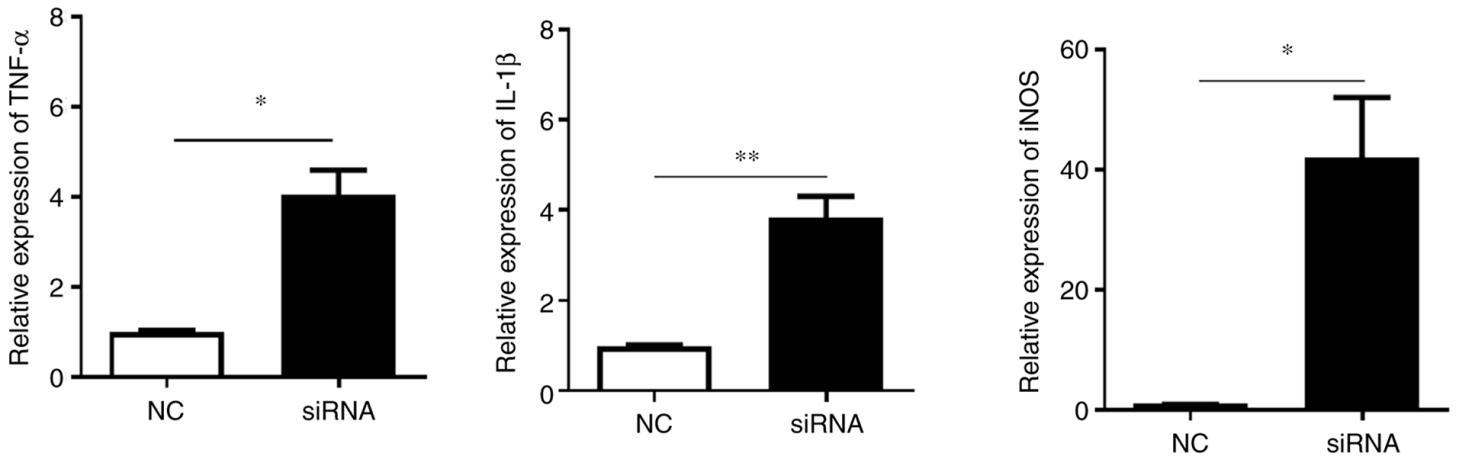

B

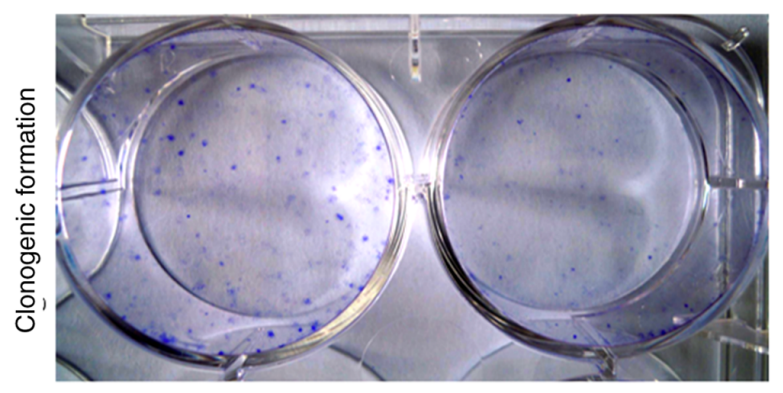

NCsi-RNA

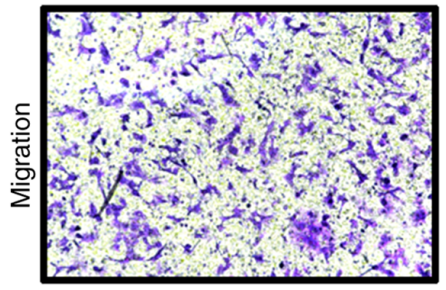

NCsi-RNA

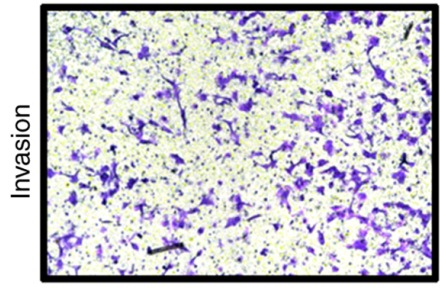

NCsi-RNA
Ly108si-RNA

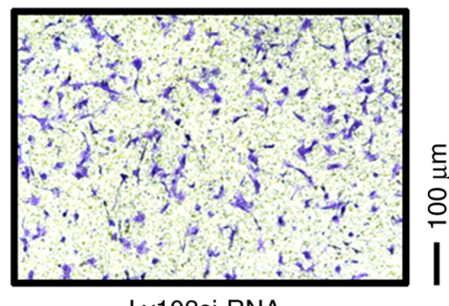

Ly108si-RNA

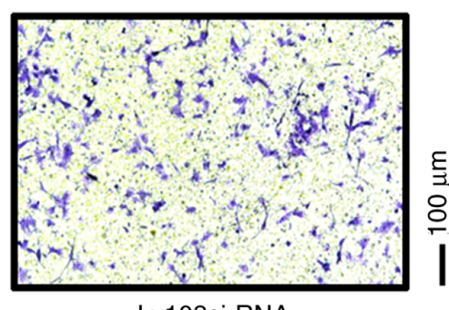

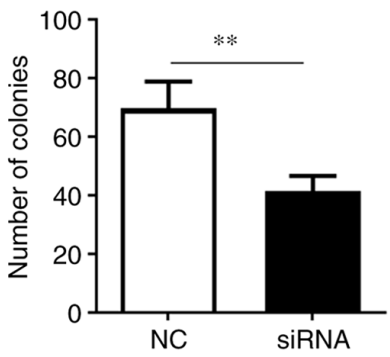
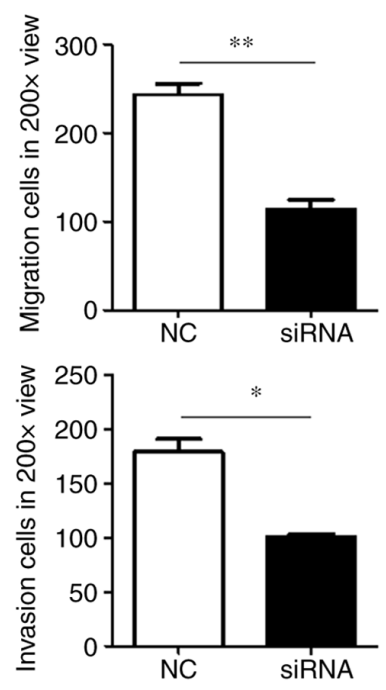

Figure 4. Ly108 promotes M2 polarization and hepatocellular carcinoma cell migration and invasion. (A) Reverse transcription quantitative PCR was performed to determine the relative expression of Ly108, arg-1, TNF- $\alpha$, IL-1 $\beta$ and iNOS in PMs, which were transfected with SLAMF6 siRNA or negative control siRNA. (B) Clonogenic formation and Transwell assays were performed to evaluate the proliferation, migration and invasion of Hepa1-6 cells. ${ }^{*} \mathrm{P}<0.05,{ }^{* *} \mathrm{P}<0.01$ and ${ }^{* * * *} \mathrm{P}<0.001$. $\mathrm{NC}$, negative control; si, small interfering; Arg-1, arginase-1; IL-1 $\beta$, interleukin 1 $1 \beta$; iNOS, inducible nitric oxide synthase; TNF- $\alpha$, tumor necrosis factor- $\alpha$.

also promoted the proliferation, migration and invasion of Hepa1-6 cells (Fig. 5D). These results indicated that Ly108 may promote $\mathrm{HCC}$ progression by inhibiting the NF- $\mathrm{KB}$ pathway. 

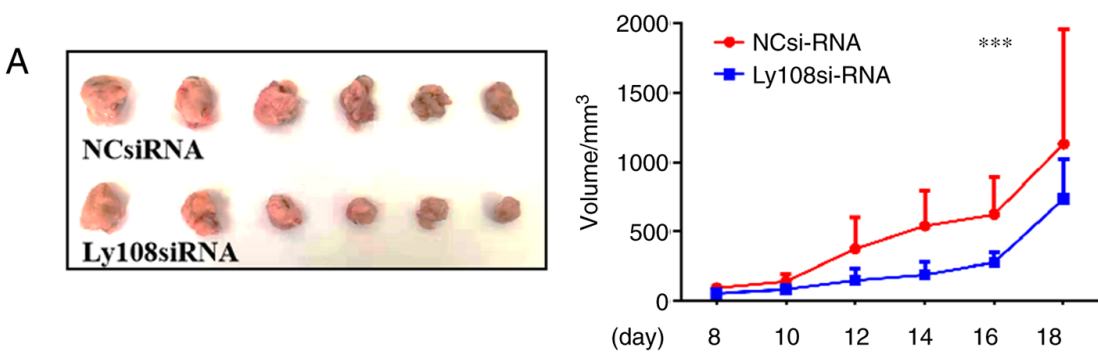

B
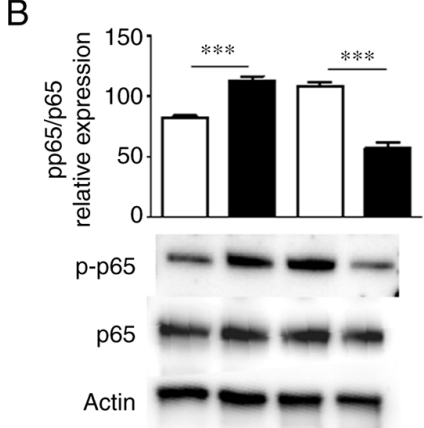

NCSiRNA

Ly108siRNA

Bay11-7082

D

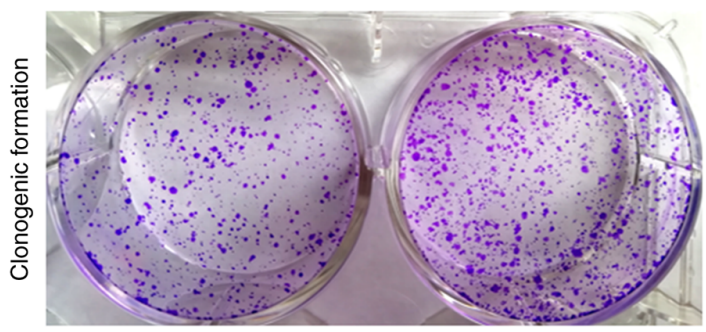

Ctr

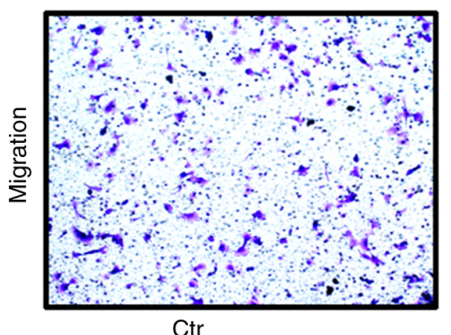

$\mathrm{Ctr}$

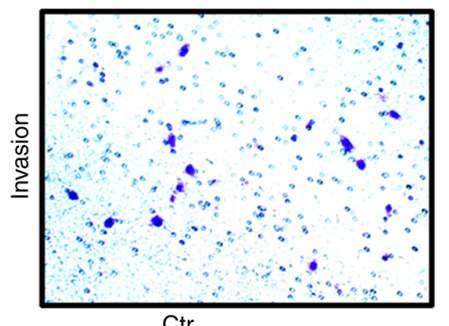

$\mathrm{Ct}$
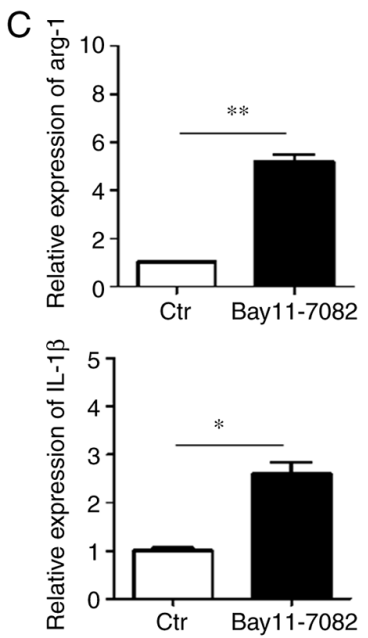

Bay11-7082
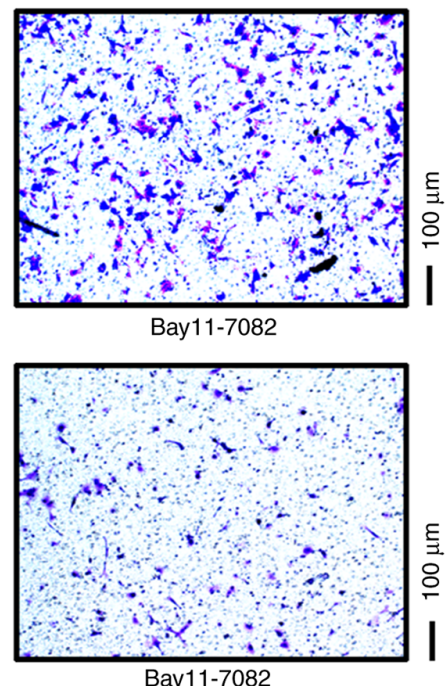
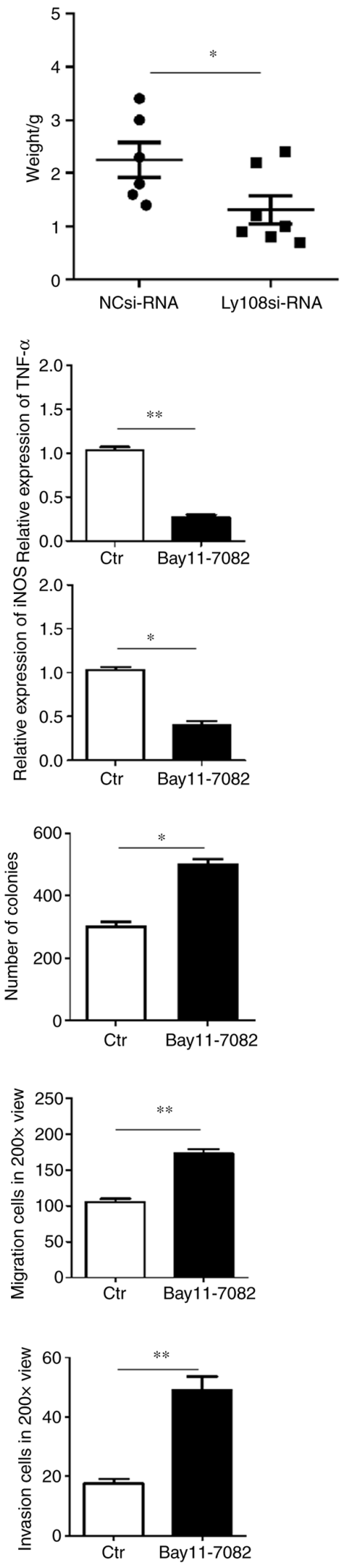

Figure 5. Ly108 promotes hepatocellular carcinoma growth by inhibiting the $\mathrm{NF}-\mathrm{kB}$ signaling pathway. (A) A murine $\mathrm{HCC}$ model was prepared ( $\mathrm{n}=6$ ) and the resulting tumors were weighed at the time of sacrifice. Tumor images are presented in the left panel, while summary data are presented in the right panel. Tumor growth was measured via tumor size, as presented in the middle panel. (B) PMs were transfected with negative control siRNA or Ly108 siRNA, with or without Bay11-7082 prior to treatment with $20 \mathrm{ng} / \mathrm{ml}$ IL-4. Western blotting was performed to determine p65 and p-p65 expression. (C) Ly108 siRNA transfection and Bay11-7082 or control DMSO stimulation prior to IL-4 induction was performed on murine PMs. Reverse transcription quantitative PCR was performed to determine the relative expression of arg-1, TNF- $\alpha, \mathrm{IL}-1 \beta$ and iNOS. (D) The macrophage culture supernatants in Figure 5C were collected and co-cultured with Hepa1- 6 cells for the transwell assays. Clonogenic formation assays were then performed. ${ }^{*} \mathrm{P}<0.05,{ }^{* *} \mathrm{P}<0.01$ and ${ }^{* * * *} \mathrm{P}<0.001$. HCC, hepatocellular carcinoma; NC, negative control; si, small interfering; Arg-1, arginase-1; IL-1 $\beta$, interleukin $1 \beta$; iNOS, inducible nitric oxide synthase; TNF- $\alpha$, tumor necrosis factor- $\alpha$; NF- $\kappa \mathrm{B}$, nuclear factor- $\kappa \mathrm{B}$; IL-4, interleukin 4; Ctr, control; p, phosphorylated; PMs, peritoneal macrophages. 


\section{Discussion}

SLAMF6 is an immune regulator that is expressed at the surface of hematopoietic cells. Previous studies have revealed that SLAMF6 is involved in numerous diseases, including systemic lupus erythematosus and lethal colitis $(25,27)$. However, the role of macrophages SLAMF6 expression in HCC progression remains unknown. The present study reported increased SLAMF6 expression levels in the HCC microenvironment, which facilitated M2-polarization and promoted HCC cell migration, invasion and proliferation.

The results from the present study suggested that there was a relationship between SLAMF6 and TAM-related tumor-promoting inflammation. SLAMF6 expression level was higher in the peripheral $\mathrm{CD}_{14}{ }^{+}$monocytes of patients with HCC compared with healthy donors and was associated with certain clinicopathological characteristics of patients. Furthermore, TAMs, also known as M2-like macrophages, exhibited higher SLAMF6 (Ly108 in mice) expression level compared with M1-like macrophages. In addition, the tumor microenvironment was determined to be the cause of high SLAMF6 expression and could also promote macrophage polarization. SLAMF6 expression in TAMs was able to promote HCC cell proliferation, migration and invasion, indicating the important role of SLAMF6 in HCC development.

After demonstrating that SLAMF6 was upregulated in the peripheral $\mathrm{CD} 14^{+}$monocytes of patients with $\mathrm{HCC}$, our study aimed to elucidate the relationship between SLAMF6 expression level and certain clinicopathological characteristics of patients with HCC, including HBV status, AFP level, the number of tumors, the lymphatic metastasis occurrence, the distant metastasis occurrence and the different stages of tumors. Only SLAMF6 expression level in the CD14 monocytes from patients with $\mathrm{HCC}$ that were $\mathrm{HBV}$ positive or whose serum AFP levels was $>100 \mathrm{ng} / \mathrm{ml}$ was significantly higher compared with the control group. This may be due to the diversity of the patients from whom blood samples were obtained. Upregulated SLAMF6 levels were also observed in the murine HCC model, which verifies this conclusion.

Macrophages are classified into two phenotypes: The classically activated M1 phenotype and the alternatively activated M2 phenotype (8). Among these, the M2-like phenotype is considered to be a TAM, serving an important role in the promotion of tumor growth and progression and accelerating HCC cell invasion and migration, thereby worsening patient prognosis $(12,13)$. The results from the present study revealed that TAMs had higher SLAMF6 expression levels compared with M1-like macrophages. Furthermore, these findings were in accordance with those obtained in human blood samples, indicating that the monocytes from patients with HCC may have a tendency towards the M2 phenotype and may therefore exhibit higher SLAMF6 expression level. The present study also demonstrated that the tumor microenvironment may facilitate macrophage SLAMF6 expression and polarization. The tumor microenvironment is extremely complex and its components could promote the expression of SLAMF6. However, this conclusion is difficult to verify. The results from the present study indicated that SLAMF6 silencing significantly inhibited the proliferation, migration and invasion of Hepa1-6 cells. Furthermore, SLAMF6 silencing could inhibit tumor growth in the murine HCC model. Taken together, these finding suggested a potential role of SLAMF6 in TAM-mediated HCC progression.

$\mathrm{NF}-\kappa \mathrm{B}$ is a signaling pathway that serves an important role in macrophage polarization (30). It has been reported that SLAMF6 regulates the function of Th2 cells via the NF- $\kappa \mathrm{B}$ pathway (29). The results from the present study demonstrated that SLAMF6 accelerated HCC growth by inhibiting the $\mathrm{NF}-\kappa \mathrm{B}$ signaling pathway.

The findings from our study suggested that increased SLAMF6 expression levels in patients with HCC may be caused by the tumor microenvironment, which promoted $\mathrm{HCC}$ development via the $\mathrm{NF}-\kappa \mathrm{B}$ signaling pathway. However, further investigation is required to determine the relationship between SLAMF6 expression and the clinicopathological characteristics of patients with HCC. Furthermore, determining why SLAMF6 expression level was upregulated in M2 macrophages and elucidating the mechanisms underlying the interactions between SLAMF6 and NF- $\mathrm{B}$ should be the aim of future studies.

\section{Acknowledgements}

Not applicable.

\section{Funding}

This study was supported by the Shandong Provincial Natural Science Foundation, China (grant no. ZR2020QH177) and the Key Research \& Development Plan of Shandong Province (grant no. 2019GSF108013).

\section{Availability of data and materials}

All data generated or analyzed during this study are included in this published article and are available from the corresponding author on reasonable request.

\section{Authors' contributions}

QM, XD and QY were responsible for the flow cytometry and molecular biology experiments. DX, ZL, YL, QJ, FG and SJ were responsible for the human sample collection. $\mathrm{ZW}, \mathrm{XC}$ and WY were responsible for the mouse model of hepatocellular carcinoma. PS designed the research and drafted the manuscript. QM and PS confirm the authenticity of all the raw data. All authors read and approved the final manuscript.

\section{Ethics approval and consent to participate}

The present study was approved by the Medical Ethics Committee of Shandong Cancer Hospital and Institute. Blood collection methods were carried out in accordance with Guidelines for the Collection of Venous Blood Specimens of China (WS/T661-2020). Informed consent was obtained from all participants. Animal experimental protocols were approved by the Animal Ethics Committee of Shandong Cancer Hospital and Institute. The methods used for the anesthesia and euthanasia of mice were carried out in accordance with the American Veterinary Medical Association Guidelines 
for the Euthanasia of Animals (2020). All methods involving animals were performed in accordance with ARRIVE (Animal Research: Reporting of In Vivo Experiments) guidelines.

\section{Patient consent for publication}

Not applicable.

\section{Competing interests}

The authors declare that there have no competing interests.

\section{References}

1. Sung H, Ferlay J, Siegel RL, Laversanne M, Soerjomataram I, Jemal A and Bray F: Global cancer statistics 2020: GLOBOCAN estimates of incidence and mortality worldwide for 36 cancers in 185 countries. CA Cancer J Clin 71: 209-249, 2021.

2. European Association for The Study of The Liver; European Organisation for Research and Treatment of Cancer: EASL-EORTC clinical practice guidelines: Management of hepatocellular carcinoma. J Hepatol 56: 908-943, 2012.

3. Pardee AD and Butterfield LH: Immunotherapy of hepatocellular carcinoma: Unique challenges and clinical opportunities. Oncoimmunology 1: 48-55, 2012.

4. Tsuchiya N, Sawada Y, Endo I, Uemura Y and Nakatsura T: Potentiality of immunotherapy against hepatocellular carcinoma World J Gastroenterol 21: 10314-10326, 2015.

5. Hernandez-Gea V, Toffanin S, Friedman SL and Llovet JM: Role of the microenvironment in the pathogenesis and treatment of hepatocellular carcinoma. Gastroenterology 144: 512-527, 2013.

6. Wan S, Zhao E, Kryczek I, Vatan L, Sadovskaya A, Ludema G, Simeone DM, Zou W and Welling TH: Tumor-associated macrophages produce interleukin 6 and signal via STAT3 to promote expansion of human hepatocellular carcinoma stem cells. Gastroenterology 147: 1393-1404, 2014.

7. Ruffell B, Affara NI and Coussens LM: Differential macrophage programming in the tumor microenvironment. Trends Immunol 33: 119-126, 2012.

8. Gordon S: Alternative activation of macrophages. Nat Rev Immunol 3: 23-35, 2003.

9. Locati M, Mantovani A and Sica A: Macrophage activation and polarization as an adaptive component of innate immunity. Adv Immunol 120: 163-184, 2013.

10. Sica A and Mantovani A: Macrophage plasticity and polarization: In vivo veritas. J Clin Invest 122: 787-795, 2012.

11. Wynn TA and Vannella KM: Macrophages in tissue repair, regeneration, and fibrosis. Immunity 44: 450-462, 2016

12. Yeung OW, Lo CM, Ling CC, Qi X, Geng W, Li CX, Ng KT, Forbes SJ, Guan XY, Poon RT, et al: Alternatively activated (M2) macrophages promote tumour growth and invasiveness in hepatocellular carcinoma. J Hepatol 62: 607-616, 2015.

13. Yao RR, Li JH, Zhang R, Chen RX and Wang YH: M2-polarized tumor-associated macrophages facilitated migration and epithelial-mesenchymal transition of HCC cells via the TLR4/STAT3 signaling pathway. World J Surg Oncol 16: 9, 2018.

14. Cannons JL, Tangye SG and Schwartzberg PL: SLAM family receptors and SAP adaptors in immunity. Annu Rev Immunol 29 665-705, 2011.

15. Veillette A: Immune regulation by SLAM family receptors and SAP-related adaptors. Nat Rev Immunol 6: 56-66, 2006.

16. Calpe S, Wang N, Romero X, Berger SB, Lanyi A, Engel P and Terhorst C: The SLAM and SAP gene families control innate and adaptive immune responses. Adv Immunol 97: 177-250, 2008.

17. Wang N, Keszei M, Halibozek P, Yigit B, Engel P and Terhorst C: Slamf6 negatively regulates autoimmunity. Clin Immunol 173 19-26, 2016.

18. Wang N, Halibozek PJ, Yigit B, Zhao H, O'Keeffe MS, Sage P, Sharpe A and Terhorst C: Negative regulation of humoral immunity due to interplay between the SLAMF1, SLAMF5, and SLAMF6 Receptors. Front Immunol 6: 158, 2015.

19. Flaig RM, Stark S and Watzl C: Cutting edge: NTB-A activates NK cells via homophilic interaction. J Immunol 172: 6524-6527, 2004.
20. Bottino C, Falco M, Parolini S, Marcenaro E, Augugliaro R, Sivori S, Landi E, Biassoni R, Notarangelo LD, Moretta L and Moretta A: NTB-A [correction of GNTB-A], a novel SH2D1A-associated surface molecule contributing to the inability of natural killer cells to kill Epstein-Barr virus-infected B cells in X-linked lymphoproliferative disease. J Exp Med 194: 235-246, 2001.

21. Marcq I, Nyga R, Cartier F, Amrathlal RS, Ossart C, Ouled-Haddou H, Ghamlouch H, Galmiche A, Chatelain D, Lamotte L, et al: Identification of SLAMF3 (CD229) as an inhibitor of hepatocellular carcinoma cell proliferation and tumour progression. PLoS One 8: e82918, 2013.

22. Bouhlal H, Ouled-Haddou H, Debuysscher V, Singh AR, Ossart C, Reignier A, Hocini H, Fouquet G, Al Baghami M, Eugenio MS, et al: RB/PLK1-dependent induced pathway by SLAMF3 expression inhibits mitosis and control hepatocarcinoma cell proliferation. Oncotarget 7: 9832-9843, 2016.

23. Wang N, Satoskar A, Faubion W, Howie D, Okamoto S, Feske S, Gullo C, Clarke K, Sosa MR, Sharpe AH and Terhorst C: The cell surface receptor SLAM controls $\mathrm{T}$ cell and macrophage functions. J Exp Med 199: 1255-1264, 2004.

24. Fouquet G, Marcq I, Debuysscher V, Bayry J, Rabbind Singh A, Bengrine A, Nguyen-Khac E, Naassila $M$ and Bouhlal H: Signaling lymphocytic activation molecules Slam and cancers: Friends or foes? Oncotarget 9: 16248-16262, 2018.

25. Chatterjee M, Kis-Toth K, Thai TH, Terhorst C and Tsokos GC: SLAMF6-driven co-stimulation of human peripheral T cells is defective in SLE T cells. Autoimmunity 44: 211-218, 2011.

26. Chatterjee M, Rauen T, Kis-Toth K, Kyttaris VC, Hedrich CM, Terhorst C and Tsokos GC: Increased expression of SLAM receptors SLAMF3 and SLAMF6 in systemic lupus erythematosus T lymphocytes promotes Th17 differentiation. J Immunol 188: 1206-1212, 2012

27. van Driel B, Wang G, Liao G, Halibozek PJ, Keszei M, O'Keeffe MS, Bhan AK, Wang N and Terhorst C: The cell surface receptor Slamf6 modulates innate immune responses during Citrobacter rodentium-induced colitis. Int Immunol 27: 447-457, 2015.

28. Yigit B, Halibozek PJ, Chen SS, O'Keeffe MS, Arnason J, Avigan D, Gattei V, Bhan A, Cen O, Longnecker R, et al: A combination of an anti-SLAMF6 antibody and ibrutinib efficiently abrogates expansion of chronic lymphocytic leukemia cells. Oncotarget 7: 26346-26360, 2016.

29. Cannons JL, Yu LJ,Hill B, Mijares LA, Dombroski D, Nichols KE, Antonellis A, Koretzky GA, Gardner K, Schwartzberg PL, et al: SAP regulates $\mathrm{T}(\mathrm{H}) 2$ differentiation and $\mathrm{PKC}$-theta-mediated activation of NF-kappaB. Immunity 21: 693-706, 2004.

30. Mussbacher M, Salzmann M, Brostjan C, Hoesel B, Schoergenhofer C, Datler H, Hohensinner P, Basílio J, Petzelbauer P, Assinger A and Schmid JA: Cell type-specific roles of NF- $\mathrm{kB}$ linking inflammation and thrombosis. Front Immunol 10: 85, 2019.

31. Crowe NY, Coquet JM, Berzins SP, Kyparissoudis K, Keating R, Pellicci DG, Hayakawa Y, Godfrey DI and Smyth MJ: Differential antitumor immunity mediated by NKT cell subsets in vivo. J Exp Med 202: 1279-1288, 2005.

32. Zhang X, Goncalves R and Mosser DM: The isolation and characterization of murine macrophages. Curr Protoc Immunol: Nov 15, 2008 (Epub ahead of print). doi: 10.1002/0471142735.im1401s83.

33. Livak KJ and Schmittgen TD: Analysis of relative gene expression data using real-time quantitative PCR and the 2(-Delta Delta C(T)). Methods 25: 402-408, 2001.

34. Sauzay C, Petit A, Bourgeois AM, Barbare JC, Chauffert B, Galmiche A and Houessinon A: Alpha-foetoprotein (AFP): A multi-purpose marker in hepatocellular carcinoma. Clin Chim Acta 463: 39-44, 2016.

35. Mantovani A, Sozzani S, Locati M, Allavena P and Sica A: Macrophage polarization: Tumor-associated macrophages as a paradigm for polarized M2 mononuclear phagocytes. Trends Immunol 23: 549-555, 2002.

This work is licensed under a Creative Commons Attribution-NonCommercial-NoDerivatives 4.0 International (CC BY-NC-ND 4.0) License. 